\title{
Neumonía necrosante causada por el serotipo 3 del Streptococcus pneumoniae a pesar de la inmunización con la VCN13 Necrotizing pneumonia caused by Streptococcus pneumoniae serotype 3 despite PCV13
}

\author{
Dra. Gülsüm Alkan ${ }^{a}$, Prof. Asist. Dra. Melike Emiroğlu ${ }^{a}$, Prof. Asoc. Dra. Hatice T. Dă̆g ${ }^{b}$, \\ Biol. Venhar Gürbüzc y Prof. Dr. Mehmet Ceyhan ${ }^{c}$
}

\begin{abstract}
RESUMEN
El Streptococcus pneumoniae es la causa más frecuente de una neumonía complicada. La neumonía neumocócica necrosante (NNN) constituye una complicación rara y relacionada con el serotipo. Los serotipos 1, 3, 14, 15, 19A y 33 fueron los más frecuentemente informados en los niños con NNN antes de la inmunización. A pesar dela práctica extendida dela vacunación, el S. pneumoniae sigue siendo la causa de las enfermedades invasivas. Aquíse informa el caso de un niño que había recibido el esquema completo con la vacuna neumocócica conjugada de 13 serotipos (VCN13) diagnosticado con NNN del serotipo 3. La progresión de la enfermedad invasiva por S. pneumoniae debe considerarse a pesar de la inmunización completa.

Palabras clave:Streptococcus pneumoniae, neumoníanecrosante, inmunización, niño.
\end{abstract}

http: / / dx.doi.org/10.5546/ aap.2019.e155

Texto completo en inglés:

http:/ / dx.doi.org/10.5546/ aap.2019.eng.e155

Cómo citar: Alkan G, Emiroğlu M, Dağı HT, Gürbüz V, Ceyhan M. Neumonía necrosante causada por el serotipo 3 del Streptococcus pneumoniae a pesar de la inmunización con la VCN13. Arch Argent Pediatr 2019;117(2):e155-e157.

\section{INTRODUCCIÓN}

El S. pneumoniae es la causa más frecuente de las enfermedades invasivas en niños, como la neumonía, la meningitis, la bacteriemia y la sepsis. Hasta la fecha, se han identificado 91 tipos capsulares diferentes según el polisacárido capsular. Los distintos serotipos

a Facultad de Medicina de Selçuk Üniversitesi, Departamento de Infectología Pediátrica, Konya, Turquía.

b. Facultad de Medicina de Selçuk Üniversitesi, Departamento de Microbiología, Konya, Turquía.

c. Departamento de Infectología Pediátrica de Hacettepe Üniversitesi, Ankara, Turquía.

Correspondencia:

Dra. Gülsüm Alkan: galkan-85@hotmail.com

Financiamiento: Ninguno.

Conflicto de intereses: Ninguno que declarar.

Recibido: 5-5-2018

Aceptado: 25-10-2018 muestran diferencias en la expresión clínica de la enfermedad. Unos 13 serotipos son responsables de más del $75 \%$ de los casos de enfermedad neumocócica invasiva (ENI) en los niños. ${ }^{1} \mathrm{La}$ vacuna neumocócica conjugada de 7 serotipos (VCN7), que incluye los serotipos 4, 6B, 9V, 14, $18 \mathrm{C}, 19 \mathrm{~F}$ y $23 \mathrm{~F}$, se introdujo en el calendario nacional de vacunación de Turquía en 2009 y luego se la reemplazó con la VCN13, que abarca los serotipos 1, 3, 5, 6A, 7F y 19A. ${ }^{2}$ En un estudio multicéntrico realizado en Turquía, las coberturas serotípicas potenciales de la VCN7, la vacuna neumocócica conjugada de 10 serotipos (VCN10) y la VCN13 fueron del $16,3 \%, 45,4 \%$ y $60 \%$, respectivamente, para el empiema paraneumónico pediátrico. ${ }^{3}$ Hacia finales de 2015, según los informes, la tasa de vacunación en Turquía con la VCN13 era del $97 \%$. A pesar de las altas tasas de vacunación y de la fuerte inmunorreacción a las vacunas conjugadas, igualmente se producen casos de complicaciones potencialmente mortales, como neumonía neumocócica necrosante (NNN) o empiema.

\section{A propósito de un caso}

Se hospitalizó a un niño de 20 meses de edad que tenía tos y fiebre de tres días de evolución y que, por lo demás, era sano. Sus antecedentes médicos y familiares no presentaban particularidades. Había recibido las dosis de la VCN13 a los 2, 4, 6 y 12 meses de edad. Durante el examen físico, tenía una temperatura corporal de $39^{\circ} \mathrm{C}$, una frecuencia de pulso de 160 latidos $/ \mathrm{min}$ y una frecuencia respiratoria de 56 respiraciones / min. Su peso era de $13 \mathrm{~kg}$ (percentilo 75) y su estatura, $90 \mathrm{~cm}$ (percentilo 90). En el examen físico, no se observaron signos que indicaran un síndrome específico. Se detectó disminución de los ruidos respiratorios en el pulmón izquierdo. El resto de los exámenes fue normal.

Datos de los análisis de laboratorio: recuento de glóbulos blancos $14,6 \times 10^{3} / \mathrm{mm}^{3}$, 
neutrófilos $65 \%$, linfocitos $10 \%$, hemoglobina $10 \mathrm{~g} / \mathrm{dl}$, plaquetas $179000 / \mathrm{mm}^{3}$, velocidad de sedimentación globular $31 \mathrm{~mm} / \mathrm{h}$, proteína C-reactiva $42 \mathrm{mg} / \mathrm{dl}$; los parámetros bioquímicos fueron normales. En la radiografía de tórax, se observó opacidad total en el pulmón izquierdo. En la tomografía computarizada (TC) de tórax, se observó consolidación extensa con neumonitis necrosante en el lóbulo inferior izquierdo y derrame pleural izquierdo moderado (Figura 1). Se insertó un tubo de drenaje torácico y se drenaron aproximadamente $250 \mathrm{ml}$ de líquido purulento. La tinción de Gram mostró diplococos grampositivos. Se inició la administración de ampicilina-sulbactam (200 mg/ $\mathrm{kg} /$ día $)$ y clindamicina ( $40 \mathrm{mg} / \mathrm{kg} /$ día) por vía intravenosa. En el cultivo del empiema, se observó desarrollo de S. pneumoniae sensible a la penicilina. La cepa se identificó como serotipo 3 mediante la reacción de Quellung con antisueros específicos para el serotipo (Statens Serum Institut, Copenhague, Dinamarca). La fiebre se resolvió en dos días pero, a pesar de la presencia del tubo de drenaje, el paciente continuó con dificultad respiratoria y derrame pleural, y el pulmón izquierdo no se vio expandido en la radiografía de tórax. A los 30 días de tratamiento, se detectaron engrosamiento pleural de $4 \mathrm{~mm}$ con atelectasia total en el pulmón izquierdo y derrame pleural denso en la TC de tórax. Después de una pleurectomía, el pulmón izquierdo se expandió rápidamente (Figura 2). Los análisis de detección inmunológica (recuento de glóbulos, recuento de linfocitos en sangre periférica, concentración de inmunoglobulina total en suero, análisis de dihidrorodamina,

FIGURA 1. Tomografía computarizada de tórax: consolidación extensa con neumonitis necrosantey derrame pleural izquierdo moderado

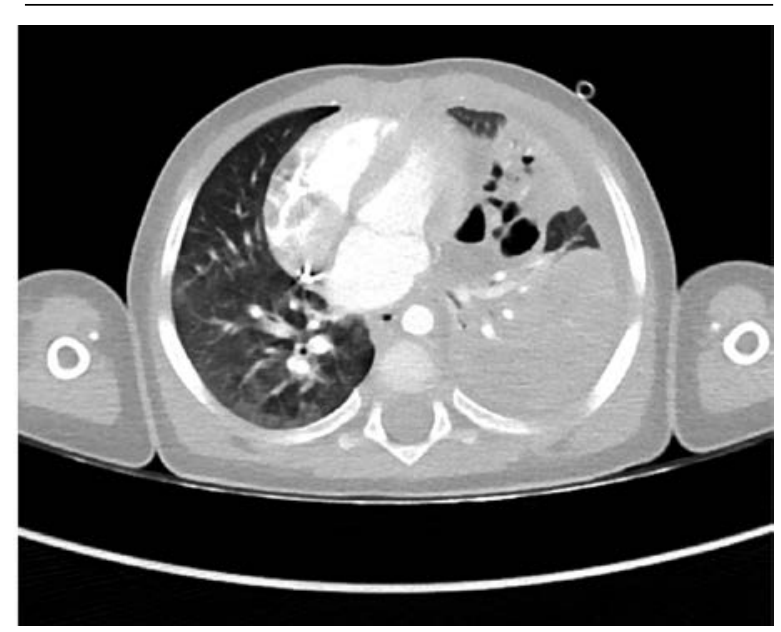

sistema del complemento) fueron normales; el análisis ELISA para la detección del virus de inmunodeficiencia humana fue negativo. La ecografía del bazo fue normal. No se detectaron cuerpos de Howell-Jolly en el frotis de sangre periférica. El valor de IgG neumocócica total fue de $2,2 \mathrm{mcg} / \mathrm{ml}$. Se completó la antibioticoterapia de seis semanas y el paciente recibió el alta. Durante el seguimiento de un año, no presentó secuelas.

\section{DISCUSIÓN}

La neumonía necrosante se caracteriza por destrucción del parénquima pulmonar, lo que produce cavidades múltiples, y a menudo está acompañada por empiema. En general, la NNN se diagnostica mediante radiografías seriadas y TC torácica. Staphylococcus aureus, Streptococcus pneumoniae y Klebsiella pneumoniae son las causas más frecuentes de la NNN. En los casos de sepsis, fiebre persistente, empiema o falta de respuesta a la antibioticoterapia se recomienda la resección quirúrgica. ${ }^{1,4-5}$

Los serotipos 1, 3, 14, 15, 19A y 33 de S. pneumoniae son los más frecuentemente informados en los niños con NNN antes de la vacunación, en especial, el serotipo $3 .{ }^{6} \mathrm{Si}$ bien no está claro el mecanismo de la necrosis con el serotipo 3, se ha planteado la hipótesis de su relación con la rápida acumulación de polisacáridos capsulares, lo que produce una carga antigénica importante y una reducción posible en las respuestas inmunitarias humorales.

A pesar de la inmunización amplia, se documentaron informes de fracaso con la

FIGURA 2. Radiografía de tórax: pulmón izquierdo expandido tras una pleurectomía

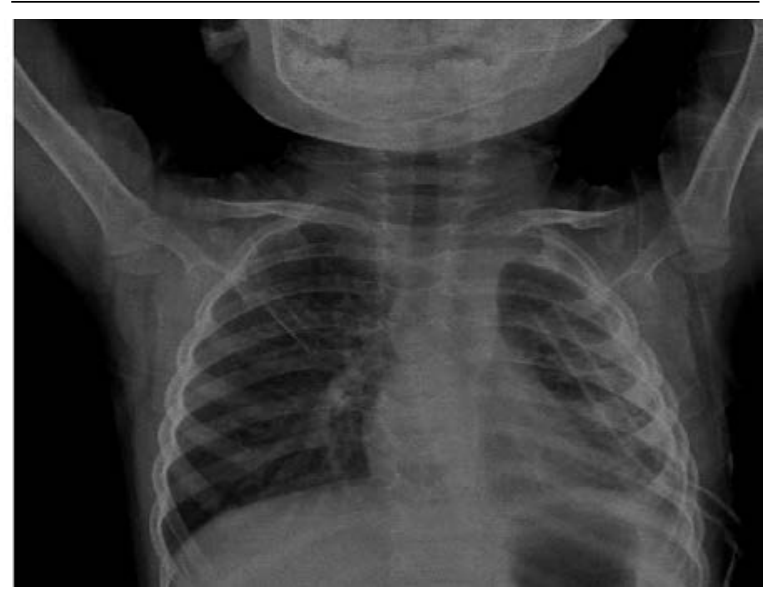


VCN13 (que incluye el serotipo 3) en casos de derrame pleural paraneumónico. Antachopoulos y col. informaron, en Grecia, casos de fracaso con la VCN13 (que incluye el serotipo 3) en pacientes con neumonía complicada. Informaron sobre cinco niños con empiema debido al serotipo 3, aunque habían recibido el esquema completo de la VCN13. Los pacientes recibieron antibioticoterapia, se les colocó un tubo torácico y se los sometió a fibrinólisis. Ninguno requirió cirugía. Las vacunas multivariadas no brindan el mismo nivel de protección con cada serotipo. En algunos estudios, se observaron respuestas a la IgG del polisacárido antineumocócico del serotipo 3 inferiores antes y después de la dosis administrada entre los 12 y los 36 meses de edad en comparación con los otros serotipos. ${ }^{8,9}$

Los neumococos se propagan mayormente hasta los pulmones desde la orofaringe. La neumonía bacteriémica representa aproximadamente el $12-16 \%$ de los casos de ENI en los niños de 2 años o menores. ${ }^{10} \mathrm{La}$ $\mathrm{VCN}$ es especialmente eficaz en las infecciones bacteriémicas. La baja protección de la vacuna en los casos de neumonía no bacteriémica podría estar relacionada con esta condición. El hemocultivo del paciente fue estéril, a pesar de que no se había usado antibiótico. Por otro lado, la resistencia del neumococo a los antibióticos es un desafío mundial. La resistencia a los antibióticos causa diferentes manifestaciones clínicas, lo que dificulta aún más su diagnóstico y tratamiento. ${ }^{11}$ Sin embargo, el cultivo de nuestro paciente mostró sensibilidad a la penicilina, por lo que la respuesta clínica no correspondió al tratamiento. En los casos de pacientes con complicaciones, se deben considerar la resistencia a los antibióticos y la inmunodeficiencia. Los casos de inmunodeficiencia primaria subyacente de una enfermedad clínica causada por el S. pneumoniae incluyen asplenia congénita, deficiencia del complemento, deficiencia de anticuerpos y mutaciones en los genes NEMO, IRAK4 o MYD88. Los análisis de detección inmunológica de nuestro paciente fueron normales. El fenotipo clínico infeccioso de la deficiencia de $I R A K-4$ y MyD88 es sumamente grave. Los signos clínicos (temperatura) y de laboratorio (concentración de PCR, recuento total de leucocitos y de neutrófilos) de inflamación suelen ser bajos, incluso en este tipo de infecciones. ${ }^{12}$ Los signos clínicos y de laboratorio de nuestro paciente no eran compatibles con deficiencia de IRAK-4 o MyD88.

Hasta donde sabemos, en la bibliografía no se ha informado ningún caso de NNN en un niño que haya completado el esquema de vacunación. La IgG neumocócica total de nuestro paciente fue positiva pero, desafortunadamente, no fue posible medir la concentración de anticuerpos específicos contra el serotipo. No es posible definir este caso como un fracaso de la vacuna con el serotipo específico.

La NNN requiere un tratamiento de mayor duración, drenaje pleural y cirugía. Independientemente de la alta tasa de vacunación, los pediatras deben estar alertas ante las complicaciones posibles de la ENI. La definición temprana de los serotipos del Streptococcus pneumoniae sería útil para determinar el enfoque terapéutico. La vigilancia activa continua es importante para determinar el fracaso de la vacuna y el diseño de vacunas nuevas.

\section{REFERENCIAS}

1. Lynch JP 3rd, Zhanel G.G. Streptococcus pneumoniae: epidemiology, risk factors, and strategies for prevention. Semin Respir Crit Care Med. 2009; 30(2):189-209.

2. Ceyhan M, Ozsurekci Y, Gürler N, Öksüz L, et al. Serotype distribution of Streptococcus pneumoniae in children with invasive diseases in Turkey: 2008-2014. Hum Vaccin Immunother. 2016; 12(2):308-13.

3. Ceyhan M, Ozsurekci Y, Gürler N, Özkan S, et al. Distribution of Streptococcus pneumoniae serotypes that cause parapneumonic empyema in Turkey. Clin Vaccine Immunol. 2013; 20(7):972-6.

4. Masters IB, Isles AF, Grimwood K. Necrotizing pneumonia: an emerging problem in children? Pneumonia (Nathan). 2017; 9:11.

5. Lu S, Tsai JD, Tsao TF, Liao PF, et al. Necrotizing pneumonia and acute purulent pericarditis caused by Streptococcus pneumoniae serotype 19A in a healthy 4-year old girl after one catch-up dose of 13-valent pneumococcal conjugate vaccine. Paediatr Int Child Health. 2016; 36(3):235-9.

6. Tsai YF, Ku YH. Necrotizing pneumonia: a rare complication of pneumonia requiring special consideration. Curr Opin Pulm Med. 2012; 18(3):246-52.

7. Bender JM, Ampofo K, Korgenski K, Daly J, et al. Pneumococcal necrotizing pneumonia in Utah: does serotype matter? Clin Infect Dis. 2008; 46(9):1346-52.

8. Diawara I, Zerouali K, Elmdaghri N, Abid A. A case report of parapneumonic pleural effusion caused by Streptococcus pneumoniae serotype 19A in a child immunized with 13-valent conjugate pneumococcal vaccine. BMC Pediatr. 2017; 17 (1):114.

9. Antachopoulos C, Tsolia MN, Tzanakaki G, Xirogianni A, et al. Parapneumonic pleural effusions caused by Streptococcus pneumoniae serotype 3 in children immunized with 13-valent conjugated pneumococcal vaccine. Pediatr Infect Dis J. 2014; 33(1):81-3.

10. Centers of Disease Control and Prevention. Pneumococcal Diseases. Clinical Features. Atlanta, 2017. [Fecha de acceso: 6 de noviembre de 2018]. Disponible en: https: / / www. cdc.gov/pneumococcal/clinicians / clinical-features.html

11. Tanır Basranoglu S, Karadag Oncel E, Aykaç K, Ozsurekci $Y$, et al. Invasive pneumococcal disease: from a tertiary care hospital in the post-vaccine era. Hum Vaccin Immunother. 2017; 13(4):962-4.

12. Picard $C$, von Bernuth $H$, Ghandil $P, C$ hrabieh $M$, et al. Clinical features and outcome of patients with IRAK-4 and MyD88 deficiency. Medicine (Baltimore). 2010; 89(6):403-25. 